\title{
Analysis of the Comprehensive Evaluation Model of Enterprise Technological Innovation Ability Based on Improved Genetic Algorithm
}

\author{
Kuizhen Rong \\ Business School, Nanjing University, Nanjing, Jiangsu 210093, China \\ Correspondence should be addressed to Kuizhen Rong; dg1902051@smail.nju.edu.cn
}

Received 9 December 2021; Revised 28 December 2021; Accepted 30 December 2021; Published 20 January 2022

Academic Editor: Miaochao Chen

Copyright (c) 2022 Kuizhen Rong. This is an open access article distributed under the Creative Commons Attribution License, which permits unrestricted use, distribution, and reproduction in any medium, provided the original work is properly cited.

\begin{abstract}
In an environment where technology is developing rapidly, product life cycles are constantly shortening, competition is increasing, and innovation resources are easily acquired by other competitors, it is particularly important for companies to successfully implement technological innovation. It is of great significance for companies to find the weak links of their technological innovation and prioritize improvement and enhancement of their technological innovation capabilities. As the main body of the agricultural machinery industry, private agricultural machinery enterprises are playing an increasingly important role. A correct understanding of the technological innovation activities of private agricultural machinery enterprises and the establishment of a reasonable evaluation index system for technological innovation capabilities are of great significance to the technological innovation management of private agricultural machinery enterprises. In accordance with the theory of technological innovation and the characteristics of private agricultural machinery enterprises, a technical innovation evaluation index system for private agricultural machinery enterprises has been established. The indicator system starts from the concept of private agricultural machinery enterprises, analyzes the status quo and development trend of the agricultural machinery industry, and takes private agricultural machinery enterprises in Heilongjiang Province as the research object. It summarizes the status quo and characteristics of technological innovation of private agricultural machinery enterprises and then establishes the private agricultural machinery enterprises. The system starts from the aspects of innovation resource input, innovation output, technology density, innovation effect, market realization, and innovation tendency, and selects 16 specific evaluation indicators. According to the established capability, projection pursuit is adopted. The method combined with the genetic algorithm and the genetic algorithm in MATLAB and the direct search toolbox were employed to comprehensively charge the capabilities of the five sample enterprises, and the evaluation results were objective and credible.
\end{abstract}

\section{Introduction}

Agricultural machinery plays an important role in promoting agriculture. From the promulgation to the implementation of a series of central support policies, China's agricultural machinery industry has ushered in a good situation of rapid development. According to statistics from the China Agricultural Machinery Industry Association, in 2005, the production and sales of the entire agricultural machinery industry in China were booming, and economic benefits were significantly improved. The industrial output value and sales revenue of the entire industry increased by $30 \%$ and $50 \%$, respectively, over 2004 [1-5].

At present, China's agricultural machinery industry has gained new opportunities for development. From 2004 to 2006, the central government successively issued three No. 1 documents, focusing on solving the problems of "farmers, agriculture, and rural areas." The No. 1 document of the central committee in 2006 focused on promoting the construction of a new socialist countryside and regarded the development of modern agriculture as the main purpose of the construction of a new countryside. The construction of a 
new countryside will vigorously promote the development of agricultural mechanization in China and enable the agricultural machinery industry to obtain new development opportunities $[6,7]$.

In recent years, private agricultural machinery companies have played an increasingly important role. With the continuous deepening of the reform process, some stateowned and collective agricultural machinery manufacturing enterprises have closed, stopped, merged, and transferred. The adjustment of the industrial structure and product structure of the agricultural machinery industry has accelerated, which has also brought about the survival and development of private agricultural machinery enterprises. There are great opportunities and challenges. China's private agricultural machinery enterprises are developing rapidly. Based on statistics, there are 920 private agricultural machinery enterprises among the 1,469 large-scale agricultural machinery enterprises, accounting for $62.6 \%$ of the scale of enterprises, with assets accounting for $43.6 \%$ and sales revenue accounting for $63 \%$. The dominant position of private agricultural machinery enterprises has been further established [8-10].

In the market environment of world economic integration and production internationalization, China's private agricultural machinery enterprises are facing a huge impact. The influx of foreign products, technologies, and funds is conducive to the introduction of advanced technology, equipment, capital, and modern management systems from developed countries by Chinese agricultural machinery enterprises, and stimulates and promotes domestic agricultural machinery enterprises to carry out technological transformation and improve product quality. At the same time, some internationally well-known agricultural machinery groups have gradually expanded their territory in China's agricultural machinery market through the implementation of "localization" strategies, showing strong vitality [11-15].

Generally speaking, China's agricultural machinery products are about 20 years behind developed countries in terms of quality and performance. The company's product development capabilities and market capabilities are weak, and the progress of industrialization is also very slow. Although private agricultural machinery companies attach great importance to technological innovation, there is still a big gap compared with agricultural machinery companies in developed countries. Some of the specific performances are as follows: insufficient $\mathrm{R} \& \mathrm{D}$ investment, lack of scientific research talents, poor independent innovation ability, poor innovation output ability, insufficient protection of patents and know-how, low product technology density, neglect of management of technological innovation activities. With the acceleration of the "localization" process of multinational agricultural machinery groups, the problem of insufficient technical strength of private agricultural machinery enterprises has become more prominent. Therefore, applying new technologies, developing new products, and vigorously promoting technological innovation are important responsibilities that private agricultural machinery companies must shoulder [16-20].
In the domestic literature, there are many articles on enterprises' technological innovation capabilities. For instance, Shi Xiaoyan proposed the AHP method for comprehensive evaluation of technological innovation capabilities and applied network system theory. Tang Yanzhao and Zou Shangang put forward a multilayer grey evaluation method and the comparison method one by one. Some other scholars set the basic structure of the indicator system of technological innovation capability into 3 index categories and 17 index items: technological innovation input (resources), technological innovation output (efficiency), and technological innovation realization (benefit), and adopted the "Linear weighted sum method" for quantitative evaluation. Zheng Chundong, He Jinsheng, and Chen Tong use a comprehensive index method to evaluate the corresponding content. $\mathrm{Bi}$ Jianguo decomposes technological innovation capabilities into six elements: talent quality, product structure, operating mechanism, technology development, market adaptation, and award-winning honors, and uses the basic methods of set, weight, and fuzzy mathematics, and summarizes it with a set of relatively quantitative indicator systems. Zhou Yuping used the AHP method to quantitatively transform many complex and ambiguous problems, and at the same time, applied BP neural network software for consistency testing, which simplifies the calculation and improves the accuracy of measuring the enterprise's technological innovation capabilities. Ma Ning and Guan Jiancheng put forward the audit content and audit benchmarks of the capabilities and put forward the audit benchmarks in the form of "scoring cards." Mei Xiaoan and Peng Junwu put forward the weak index multiple method. Hu Enhua used the methods of set, weight, and fuzzy mathematics to construct an index system and put forward a method for comprehensive evaluation. Kang Kai, Xing Jing, Zhang Huiyun, and Qi Lili used fuzzy cluster analysis to establish a multilevel decomposition and evaluation model for enterprise technological innovation capabilities. Lu Juchun and Korean use the osculating value method. Lu Huaibao, Feng Yingjun, Qu Shiyou, Xu Bocheng, and Chen Jinxia proposed a secondary relative evaluation method for measuring the technological innovation capability of enterprises. First, the state of the comprehensive index was measured. The BCC model is employed to evaluate the secondary relative evaluation value. Li Qi uses the fuzzy comprehensive evaluation method. Li Huanghua, Hu Yunquan, and others used the principal component projection method to evaluate the technological innovation capabilities of enterprises in the old industrial base [21-25].

It can be seen from the abovementioned literature that the comprehensive evaluation methods of enterprises' technological innovation capabilities are diversified. The following is a simple comparison of the evaluation methods of enterprise technological innovation capabilities. The fuzzy comprehensive evaluation method (as shown in Figure 1) is a method that applies the principle of fuzzy relationship synthesis to comprehensively evaluate the status of the 


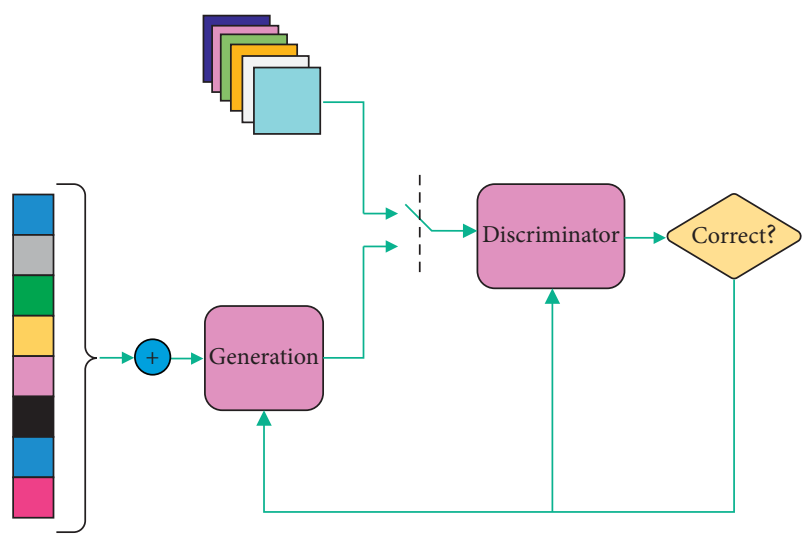

Figure 1: Principles of neural networks.

subject's membership level from multiple factors. It is widely used. Fuzzy evaluation has good adaptability when dealing with evaluation problems with more qualitative indicators, and it has strong applicability to indicators with subjective evaluation factors. It can be applied to type recognition systems, expert evaluation systems, and multiobjective social evaluation systems with comment sets.

DEA is a multiobjective evaluation method for studying multiple input and output problems. The data envelopment analysis (DEA) method needs to meet five prerequisites when it is applied; that is, convexity, cone, invalidity, primitiveness, and minimality are satisfied between the output and the input. It builds a model based on the principles of operations research, based on the largest (small) possible boundary, and selects the best possible solution in the reachable area. OEA analysis is mainly applicable [26, 27].

The combined weighting comprehensive evaluation model has actually been developed. It uses fuzzy mathematics and nonlinear optimization theory to establish mathematical models.

Grey relational analysis is essentially to construct a mapping. The correlation set of the analysis system is used to solve the correlation degree difference between the elements. Grey relational analysis is mainly used to analyze the degree of influence between factors and the measurement of the factor's contribution to the main behavior.

Factor analysis is an important multivariate statistical method. The core is to select a few random variables that can control the original variables. The factor analysis method is mainly applicable to the index difference analysis and index classification of the multiobjective evaluation system. Principal component analysis can be regarded as a special case of factor analysis.

AHP is a universally practical multicriteria decisionmaking (evaluation) method combining qualitative and quantitative elements. Its drawbacks are concentrated in the following: the index system is not comprehensive and objective; the determination of the evaluation index weight lacks theoretical basis and has technological innovation ability that changes with the outside world. Therefore, the accuracy and motivation of the evaluation results are limited.
Artificial neural network (ANN) is a frontier hot spot in nonlinear science. The advantage of using the neural network model in an enterprise is that the artificial neural network model is determined after the participating sample training, which can be iterated repeatedly according to the optimal algorithm criteria and continuously adjust the neural network structure until it reaches a relatively stable state; it can make the system error reach any accuracy requirements and convergence; further time learning and dynamic tracking evaluation can be carried out. However, in the absence of a large number of training samples, it is difficult to use neural network models.

The genetic algorithm is shown in Figure 2. It was first proposed in the United States in 1962. It can be used with the help of computer programs. It regards a family of randomly generated feasible solutions as the parent population, and the fitness function (objective function or some deformation of it) as a measure of the ability of the parent individual to adapt to the environment. After selection and crossing, the offspring individuals are generated, and the latter is then mutation, the survival of the fittest. Such repeated evolutionary iterations, continuously improve the adaptability of individuals, and excellent individuals are constantly approaching their best points. The projection pursuit method is a multivariate data analysis algorithm proposed by Friedman in the 1970s. This method is a statistical method for dealing with complex problems with multiple factors. It reasonably converts multiple evaluation index problems into a single comprehensive evaluation index, that is, comprehensive evaluation in one-dimensional space. This method overcomes the shortcomings of the general evaluation process, such as the lack of theoretical basis and calculation formulas in the determination of weights, and solves the problem that the model is affected by subjective factors. The projection pursuit method is a high-dimensional data analysis method that can be used for exploratory analysis and deterministic analysis and is especially suitable for comprehensive evaluation of multi-index and nonlinear problems. This paper evaluates the technological innovation ability of private agricultural machinery enterprises [28, 29].

\section{Evaluation Model}

Projection pursuit (PP) is a nonparametric high-dimensional data processing and exploratory data analysis method developed by the international statistical community in the mid-1970s. It is a dimensionality reduction (regression) technology (Hall. P, 1959). In the late 1960s and early 1970s, Krusca first proposed the projection pursuit method. Switze et al. successfully applied projection pursuit technology to the study of fossil classification problems in combination with numerical calculation methods. In 1974, Friedman and Tukey carried out an in-depth study on the projection pursuit method and clearly proposed the projection pursuit idea. That is, the sample data and the low-dimensional structure obtained by the projection are defined by the projection index. Use a computer to find the maximum projection direction (or plane) of the projection index (or several), give the data projection on a straight line (or plane), 


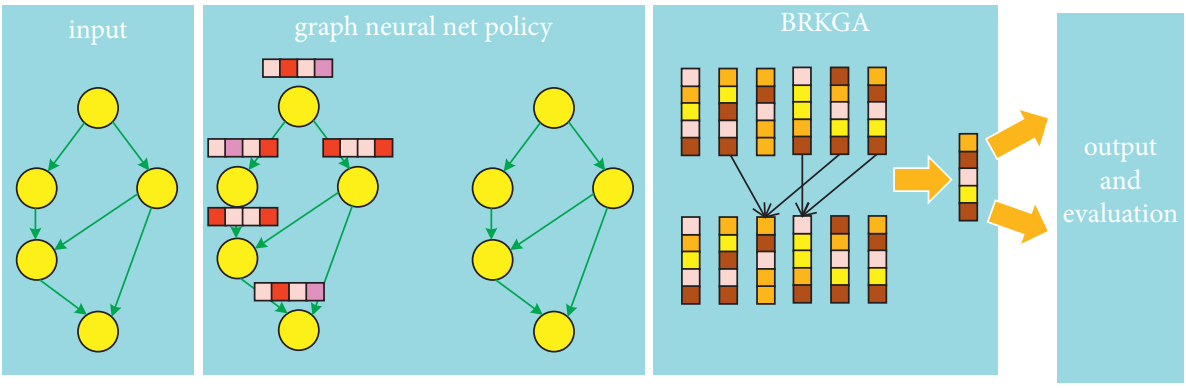

FIgURE 2: Genetic algorithm.

display it by the computer image system, and then directly judge with the naked eye the data structure characteristics. On this basis, one-dimensional and two-dimensional projection indexes of projection pursuit cluster analysis are constructed, which has made a great contribution to the development of projection pursuit technology. Later, projection pursuit methods have made major breakthroughs in theoretical research and practical applications and have successively formed and a large number of projection pursuit methods. Technology research results. In 1985, Huber published a comprehensive academic paper on projection pursuit, systematically expounding the projection pursuit theory, marking the formal formation of the projection pursuit theory. This article uses the projection pursuit method to evaluate the technological innovation ability of private agricultural machinery enterprises. It reasonably converts multiple evaluation index problems into a single comprehensive evaluation index form, that is, comprehensive evaluation in one-dimensional space.

The traditional multivariate obeys the normal distribution, but many practical problems do not satisfy the normal assumption and need to be solved by robust or nonparametric methods. In response to the robustness of high-dimensional sum estimation, the international statistical community has proposed the exploratory data analysis method (EDA) of "directly from examining the data-analyzing and simulating data by computer-designing software program verification" in the past 20 years, and projection pursuit (projection pursuit, PP) is one of the effective calculation methods.

A very important method of thinking in multivariate analysis is to use "dimensionality reduction" technology to project points in high-dimensional space to low-dimensional space in an appropriate way; that is, to replace highdimensional vectors with low-dimensional vectors approximately, and then in low-dimensional space. Then, proceed to group identification. The basic idea of PP is to project high-dimensional data onto a low-dimensional (1-3dimensional) subspace, looking for a projection that can reflect the structure or characteristics of the original highdimensional data, which is called an "interesting" projection, and then, through analysis and study, the projection data to achieve the purpose of understanding the original data set. In $\mathrm{PP}$, the degree of interest is reflected by the "projection index" function. It is especially suitable for analyzing and processing nonlinear, non-normal, and high-dimensional data. This method overcomes the lack of a theoretical basis and calculation formula in the determination of weights in the general evaluation process and solves the problem that the model is affected by subjective factors. The projection pursuit method is a high-dimensional data analysis method that can be used for exploratory analysis and deterministic analysis and is especially suitable for comprehensive evaluation of multi-index and nonlinear problems.

The general steps of the PP method for comprehensive evaluation are as follows.

2.1. Normalization of the Sample Evaluation Index Set. The sample set of each indicator value is as follows:

$$
\left\{x^{*}(i, j) \mid i=1 \sim n, j=1-p\right\},
$$

where $x^{*}(i, j)$ is the $j$-th index value of the $i$-th sample and $n$ and $p$ are the number of samples (sample capacity) and the number of indexes, respectively.

For the bigger and smaller, the better the index, which is as follows:

$$
\begin{aligned}
& x(i, j)=\frac{x^{*}(i, j)-x_{\min }(j)}{x_{\max }(j)-x_{\min }(j)}, \\
& x(i, j)=\frac{x_{\max }(j)-x^{*}(i, j)}{x_{\max }(j)-x_{\min }(j)},
\end{aligned}
$$

where $x^{*}(i, j)$ is the $j$-th index value of the $i$-th sample; $x_{\max }(j)$ is the maximum value of the $J$-th index; $x_{\min }(j)$ is the minimum value of the $J$-th index; and $x(i, j)$ is the normalized sequence of index feature values.

2.2. Constructing the Projection Index Function. The PP method for constructing the projection index function is as follows:

$$
z(i)=\sum_{j=1}^{p} a(j) x(i, j) .
$$

Here, $a$ is the unit length vector.

$$
z(i)=\sum_{j=1}^{p} a(j) x(i, j) .
$$

where $Q(a)$ is the projection index function. 
2.3. Optimizing the Projection Index Function. Maximize the objective function:

$$
\text { Max: } Q(a)=S_{z} \cdot D_{z} \text {. }
$$

Restrictions are given as follows:

$$
\text { s.t: } \sum_{j=1}^{p} a^{2}(j)=1 \text {. }
$$

This article intends to use genetic algorithms to optimize it.

2.4. Comprehensive Evaluation. Substituting the obtained best projection direction into (3), the projection value of each sample point can be obtained. The size of the projection value in this article is the strength of technological innovation ability.

\section{Improved Genetic Algorithm}

3.1. Traditional Genetic Algorithm. This method simulates the natural evolution of organisms by natural selection and crossover and can effectively solve more complex problems with the help of computer programs. Nonlinear combination problems and multiobjective function optimization problems. It regards a family of randomly generated feasible solutions as the parent population and uses the fitness function (objective function or some deformation of it) as a measure of the ability of the parent individual to adapt to the environment. After selection and hybridization, the offspring individuals are generated, and the latter is then mutation, the survival of the fittest. Such repeated evolutionary iterations continuously improve the adaptability of individuals, and excellent individuals are constantly approaching their best points.

Better results can be obtained by using genetic algorithms. For an optimization problem to find the maximum value, the following formula is used:

$$
\left\{\begin{array}{l}
\max f(X), \\
\text { s.t. } X \in R, \\
R \subseteq U
\end{array}\right.
$$

In optimization problems, there are many types of objective functions and constraints. Because traditional methods have too high requirements on the continuity and divergence of the data itself, which limits the scope of their application, genetic algorithms can simply overcome these shortcomings.

Because of their simplicity, ease of implementation, and robustness, genetic algorithms have been widely used in optimization and machine learning. But it also has shortcomings and limitations in practical applications, mainly in the problem of premature convergence and the problem of local search ability of genetic algorithms.

3.2. Improved Genetic Algorithm. Artificial neural network (ANN) is a frontier hot spot in nonlinear science. The advantage of using the neural network model in an enterprise is that the artificial neural network model is determined after the participating sample training, which can be iterated repeatedly according to the optimal algorithm criteria and continuously adjust the neural network structure until it reaches a relatively stable state; it can make the system error reach any accuracy requirements and convergence; further time learning and dynamic tracking evaluation can be carried out. However, in the absence of a large number of training samples, it is difficult to use neural network models.

To illustrate the improved genetic algorithm, it can be seen in the following equations:

$$
\begin{aligned}
& P_{c}=\frac{k_{1}\left(f_{\max }-f^{\prime}\right)}{f_{\text {max }}-f}, f^{\prime} \geq \bar{f}, \\
& P_{c}=k_{2}, f^{\prime}<\bar{f}, \\
& P_{m}=\frac{k_{3}\left(f_{\max }-f^{\prime}\right)}{f_{\max }-f}, f^{\prime} \geq \bar{f}, \\
& P_{m}=k_{4}, f^{\prime}<\bar{f},
\end{aligned}
$$

where $f_{\max }$ is the maximum fitness of the current population; $f$ is the fitness of a certain body; $f$ is the average fitness of the group; $k 1, k 2$ are the constant between $[0,1]$ and $k 1<k 2$; and $k 3, k 4$ are the constant between $[0.001,0.01]$ and $k 3>k 4$.

It can be seen from the abovementioned formula that if the individual is poor (fitness value < average fitness value), give it a larger crossover rate and a smaller mutation rate; if the individual is better (fitness value $>$ average fitness value), the individual is assigned the corresponding crossover rate and mutation rate according to its degree of excellence. This crossover and mutation rate adjustment method has a strong global search ability in the early stages of evolution. As evolution progresses, the global search ability gradually weakens, and the local search ability gradually increases. This improvement measure helps protect good individuals, facilitates finding the best overall advantage, and prevents "premature maturity" from appearing.

\subsection{Application of Improved Genetic Algorithm Solution Steps.} This method simulates the natural evolution of organisms by natural selection and crossover and can effectively solve more complex problems with the help of computer programs. There are both nonlinear combination and multiobjective function optimization problems. The steps of applying the improved genetic algorithm are shown in the flowchart in Figure 3. The specific process is as follows:

(1) Initialization: in this paper, real number encoding is used to randomly generate $m$ (that is, the number of specific parameters) real numbers, which are arranged together to form an individual, and $\mathrm{N}$ such individuals are continuously generated to form an initial group.

(2) Calculate fitness value: each individual uses the calculated $D i$ as the objective function value. The smaller the $D i$, the greater the individual fitness. Therefore, individual fitness can be calculated by 


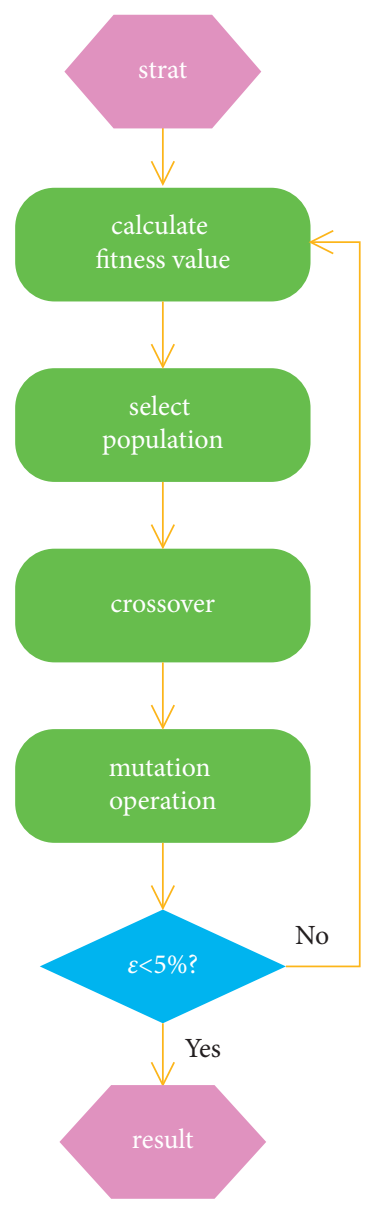

FIgURE 3: Flow chart of improved genetic algorithm.

$$
f_{i}=\frac{1}{D_{i}}
$$

(3) Select the population according to the roulette method.

(4) Perform a crossover calculation based on the current crossover probability.

(5) Perform a mutation calculation based on the current mutation probability.

(6) If the termination condition is met, the iteration is terminated, otherwise it returns to (2) the iterative solution. The termination condition can be the maximum number of cycles (usually 30 to 200) or the minimum error threshold.

(7) Output the result.

\section{Instance Verification}

In order to find the technological innovation gap between a certain enterprise and other enterprises, a horizontal comparison of the technological innovation capabilities of each enterprise can be carried out. The collection of raw data is a very critical step. The accuracy of the raw data greatly affects the scientificity and rationality of the entire index system. Refer to the "Oslo Handbook" for the guidance of collecting technological innovation data, according to the evaluation index system of technological innovation ability mentioned above, combined with the characteristics of private agricultural machinery enterprises. This article mainly adopts data collection methods such as visits and surveys, questionnaires, and emails. In order to grasp the company's technological innovation trends in more detail, the survey has extensively collected the company's product introduction, annual summary, development plan, and other related materials. For the data that the company has not counted, the expert scoring method is applied, and the chief engineer or general manager of the company is directly asked. The statistics of the collected data are in accordance with the calculation methods of the indicators described previously.

Before applying the projection pursuit method for calculation, it is necessary to standardize the above raw data. Since each index belongs to the larger the better, the abovementioned raw data is standardized.

Using the MATLAB algorithm toolbox can easily optimize the projection index function, eliminating the cumbersome programming in other languages (Figures 4 and 5).

Through the algorithm and direct search, the optimization function in the toolbox always finds the minimum value of the objective function or fitness function. If you need the maximum value of the function $f(x)$, you can convert it to the function $g(x)=f(x)$. Properly modify the algorithm parameters, select the population scale as 100 , the population type as DoubleVector (double-precision vector), and the Fitnesscaling (fitness ratio parameter) as Rank (weigh the individual's pros and cons according to the order of fitness values), and set the parameters Selectionfunction (selection function) as Uniform (uniform), the number of elites in the regeneration function is set to 5 , the variation function is selected as Uniform (uniform), and the rest are set to default values. Click Start in the Runsolve column to run and get the result.

According to the abovementioned method, use the genetic algorithm toolbox in MATLAB to calculate and get the best projection direction. ' $=\left[\begin{array}{lllll}0.0777 & 0.3952 & 0.0920 & 0.4445\end{array}\right.$ $\begin{array}{llllllll}0.2662 & 0.1264 & 0.0473 & 0.0453 & 0.0300 & 0.2239 & 0.1725 & 0.2157\end{array}$ $\left.\begin{array}{llll}0.4942 & 0.2138 & 0.3158 & 0.1538\end{array}\right]$.

Will. 'Substitute $z={ }^{\prime *} x$, get the projected value $z=\left[\begin{array}{lllll}2.5627 & 0.4360 & 1.0460 & 0.6536 & 1.1191\end{array}\right]$, arrange $z$ in descending order, and get the technological innovation ability of each enterprise in order: enterprise $A>$ enterprise $E>$ enterprise $C>$ enterprise $D>$ Enterprise B.

According to the best projection direction, the degree of influence of each evaluation index on the comprehensive evaluation result can be further analyzed. The value of a' into.

Sort rows to get the contribution rate of each indicator. The order number is as follows: $13,4,2,15,5,10,12,14,11$, $16,6,3,1,7,8$, and 9 , which is the new product market share, $\mathrm{R} \& \mathrm{D}$ personnel investment intensity, $\mathrm{R} \& \mathrm{D}$ expenditure investment intensity, product market, $J$ nonown rate, patent 


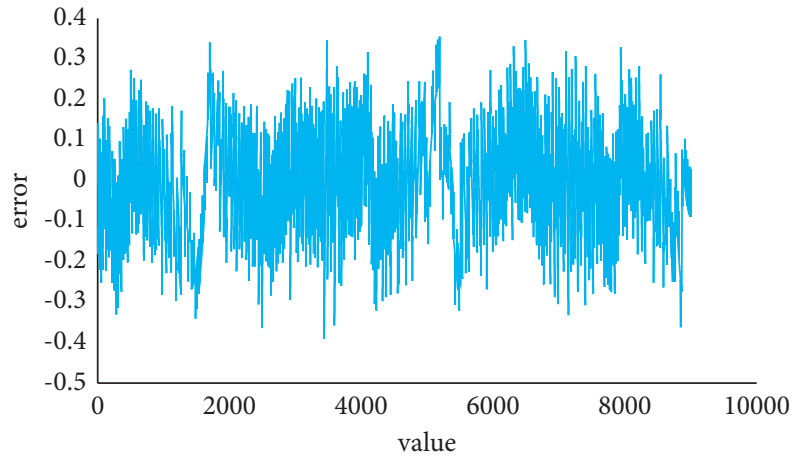

FIGURE 4: Error at different values.

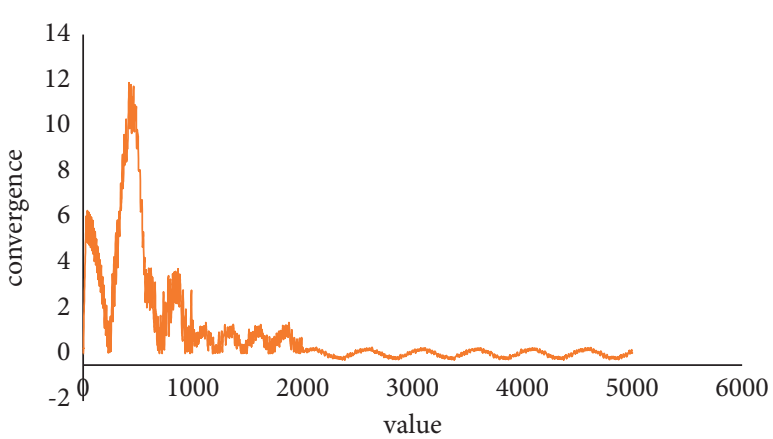

Figure 5: Convergence at different values.

and proprietary technology ownership, modern manufacturing technology utilization rate, new product sales revenue/product sales revenue, enterprise full-time marketing, total number of personnel/employees, standardized work level, annual average growth rate of $\mathrm{R} \& \mathrm{D}$ investment intensity, independent innovation product rate, process innovation investment intensity, total $\mathrm{R} \& \mathrm{D}$ investment, $R \& D$ success rate, advanced level of equipment, technical level of workers, and work quality. The best individual obtained, that is, the contribution rate of each indicator is shown in Figure 6.

In order to find the technological innovation gap between a certain enterprise and other enterprises, a horizontal comparison of the technological innovation capabilities of each enterprise can be carried out. This paper divides the technological innovation capability of private agricultural machinery enterprises into six aspects: innovation resource input, innovation output, technology density, innovation effect, market realization, and innovation tendency. In order to more simply and clearly reflect the strengths and weaknesses of various aspects of the enterprise, the technical innovation index system was slightly adjusted. The only indicator of innovation tendency-the average annual growth rate of $\mathrm{R} \& \mathrm{D}$ investment intensity-is classified as a dynamic indicator of innovation resource input. In order to more clearly reflect the gap between various companies, use the cloud diagram, shown in Figures 7 and 8, (based on MATLAB) for comparison.

Compared with agricultural machinery companies in developed countries, there is a big gap in technological

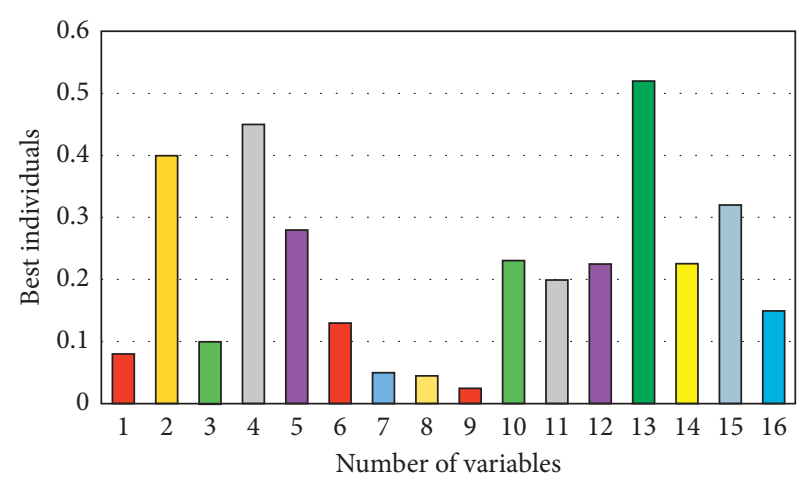

Figure 6: Best individuals.

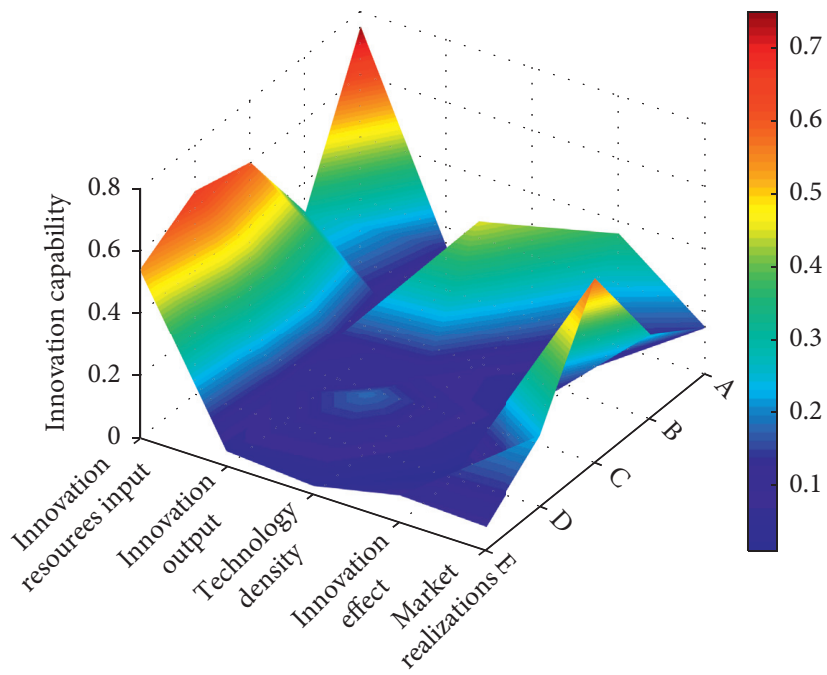

Figure 7: Gap between industries.

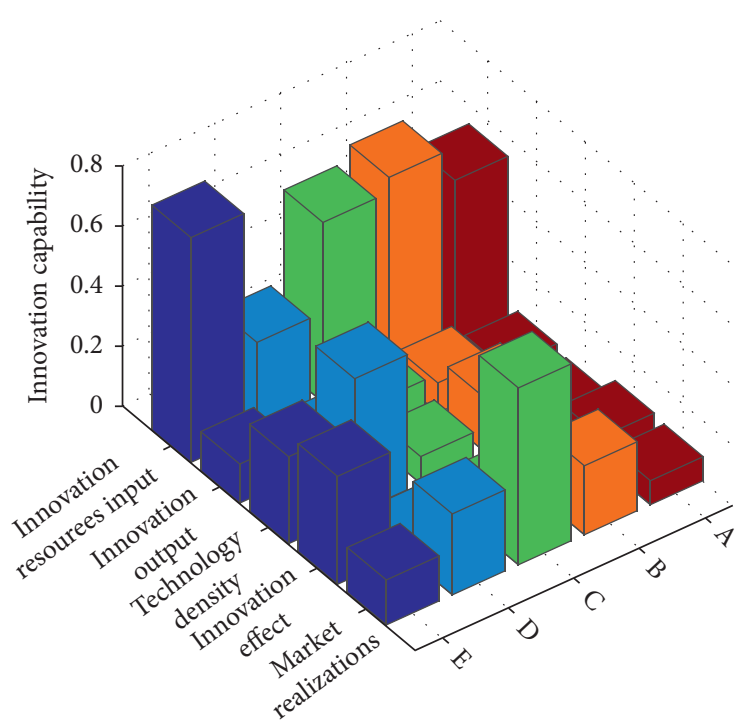

FIGURE 8: Comparison of various industries.

innovation among China's agricultural machinery companies. In order to find the technological innovation gap between a certain enterprise and other enterprises, a horizontal comparison of the technological innovation capabilities of 


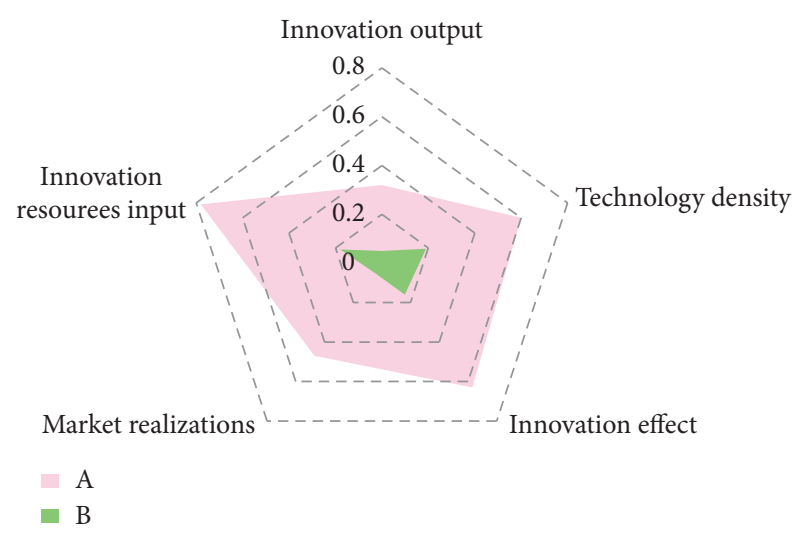

FIgURE 9: Horizontal comparison between enterprises.

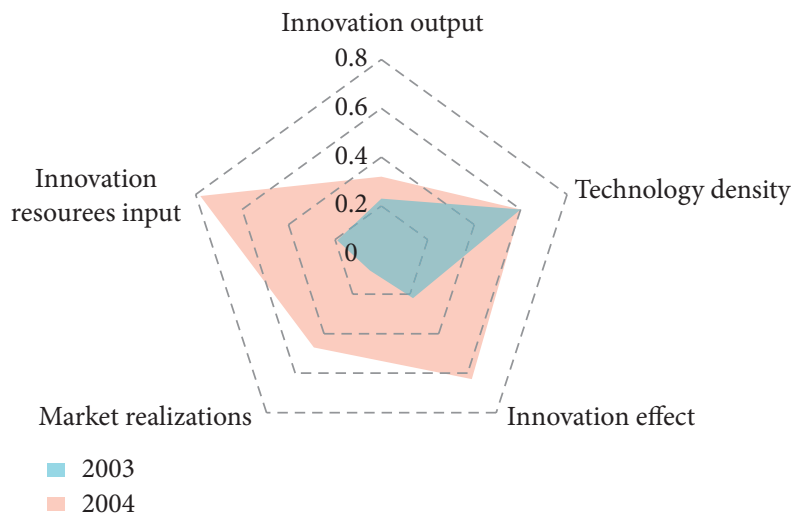

Figure 10: Vertical comparison between enterprises.

each enterprise can be carried out. For example, the comparison result of the technological innovation subcapabilities of A company and B company can be represented by radar chart 9 . It can be seen from Figure 9 that all the subcapabilities of technological innovation of $B$ company are lower than those of A company. In the same way, you can compare the technological innovation gaps between any companies. At the same time, in order to grasp the development of a certain enterprise's technological innovation activities, it is possible to make a vertical comparison of the technological innovation level of the enterprise in different years. For example, the comparison of the technological innovation level of an enterprise in 2003 and 2004 is shown in Figure 10. It can be seen from the figure that the level of technological innovation of the company in 2004 was higher than that of 2003, and the company's technological innovation activities made rapid progress.

\section{Conclusions}

Five conclusions are drawn from this paper, which are as follows:

(1) The projection pursuit method and genetic algorithm are used. Only the original observation data is used directly, without any prior assumptions about the data structure or characteristics, and human interference is eliminated, and the objectivity is good. It can make full use of the information existing in the data and can effectively process normal or non-normal data, linear or nonlinear data, with good stability and high accuracy.

(2) Selection of projection pursuit parameters. The construction of projection index is very important, it reflects the projection rule. There is no fixed form and standard for the construction of the projection index. It can be selected flexibly according to specific problems in the application, but the constructed projection index must reflect the characteristics of the analysis problem in order to achieve the purpose of reasonable evaluation of the data sample.

(3) Selection of projection direction and other parameter optimization methods. In theory, all optimization methods can achieve the optimization of projection direction and other parameters.

(4) When using the genetic algorithm to calculate the optimal projection direction, due to the different states of the random number generator, the results of each run will be different, that is, the optimal projection direction. There will be differences, but it hardly affects the order of the projection values.

(5) Analysis of evaluation results. By comparing the obtained evaluation results with the actual technological innovation capabilities of the enterprise, it can be seen that the evaluation method is credible and scientific.

\section{Data Availability}

The data used to support the findings of this study are available from the corresponding author upon reasonable request.

\section{Conflicts of Interest}

The author declares no conflicts of interest.

\section{References}

[1] Y. Yu, C. Yang, Q. Deng, T. Nyima, S. Liang, and C. Zhou, "Memristive network-based genetic algorithm and its application to image edge detection," Journal of Systems Engineering and Electronics, vol. 32, no. 5, pp. 1-9, 2021.

[2] Y. Ishida and S. Hashimoto, "Asymmetric characterization of diversity in symmetric stable marriage problems: an example of agent evacuation," Procedia Computer Science, vol. 60, no. 1, pp. 1472-1481, 2015.

[3] P. Zoha and R. Kaushik, "Image edge detection based on swarm intelligence using memristive networks," IEEE Trans. on CAD of Integrated Circuits and Systems, vol. 37, no. 9, pp. 1774-1787, 2018.

[4] J. Pais, "Random matching in the college admissions problem," Economic Theory, vol. 35, no. 1, pp. 99-116, 2018.

[5] J. J. Jung and G. S. Jo, "Brokerage between buyer and seller agents using constraint satisfaction problem models," Decision Support Systems, vol. 28, no. 4, pp. 291-384, 2020. 
[6] Y. Liu and K. W. Li, "A two-sided matching decision method for supply and demand of technological knowledge," Journal of Knowledge Management, vol. 21, no. 3, 2017.

[7] J. Byun and S. Jang, "Effective destination advertising: matching effect between advertising language and destination type," Tourism Management, vol. 50, no. 10, pp. 31-40, 2015.

[8] A. N. Nagamani, S. N. Anuktha, N. Nanditha, and V. K. Agrawal, "A genetic algorithm-based heuristic method for test set generation in reversible circuits," IEEE Transactions on Computer-Aided Design of Integrated Circuits and Systems, vol. 37, no. 2, pp. 324-336, 2018.

[9] C. Koch and S. P. Penczynski, "The winner's curse: conditional reasoning and belief formation," Journal of Economic Theory, vol. 174, pp. 57-102, 2018.

[10] C. K. Karl, "Investigating the winner's curse based on decision making in an auction environment," Simulation \& Gaming, vol. 47, no. 3, pp. 324-345, 2016.

[11] D. Ettinger and F. Michelucci, "Creating a winner's curse via jump bids," Review of Economic Design, vol. 20, no. 3, pp. 173-186, 2016.

[12] J. A. Brander and E. J. Egan, "The winner's curse in acquisitions of privately-held firms," The Quarterly Review of Economics and Finance, vol. 65, pp. 249-262, 2017.

[13] Z. Palmowski, "A note on var for the winner's curse," Economics/Ekonomia, vol. 15, no. 3, pp. 124-134, 2017.

[14] B. R. Routledge and S. E. Zin, "Model uncertainty and liquidity," Review of Economic Dynamics, vol. 12, no. 4, pp. 543-566, 2009.

[15] D. Easley and M. O'Hara, “Ambiguity and nonparticipation: the role of regulation," The Review of Financial Studies, vol. 22, no. 5, pp. 1817-1843, 2019.

[16] P. Klibano, M. Marinacci, and S. Mukerji, "A smooth model of decision making under ambiguity," Econometrica, vol. 73, no. 6, pp. 1849-1892, 2005.

[17] Y. Halevy, "Ellsberg revisited: an experimental study," Econometrica, vol. 75, no. 2, pp. 503-536, 2017.

[18] D. Ahn, S. Choi, and D. Gale, "Estimating ambiguity aversion in a portfolio choice experiment," Working paper, vol. 5, no. 2, pp. 195-223, 2019.

[19] T. Hayashi and R. Wada, "Choice with imprecise information: an experimental approach," Theory and Decision, vol. 69, no. 3, pp. 355-373, 2010.

[20] K. Zima, E. Plebankiewicz, and D. Wieczorek, "A SWOT analysis of the use of bim technology in the polish construction industry," Buildings, vol. 10, no. 1, 2020.

[21] P. Sun, B. Liu, and T. Sun, "Injury status and strategies of female 7-a-side rugby players in Anhui Province," Sports Boutique, vol. 38, no. 03, pp. 72-74, 2019.

[22] P. Guild, M. R. Lininger, and M. Warren, "The association between the single leg hop test and lower-extremity injuries in female athletes: a critically appraised topic," Journal of Sport Rehabilitation, vol. 30, no. 2, pp. 1-7, 2020.

[23] U. G. Inyang, E. E. Akpan, and O. C. Akinyokun, "A hybrid machine learning approach for flood risk assessment and classification," International Journal of Computational Intelligence and Applications, vol. 19, no. 2, Article ID 2050012, 2020.

[24] Q. Liu, S. Du, B. Wyk, and Y. Sun, "Double-layer-clustering differential evolution multimodal optimization by speciation and self-adaptive strategies," Information Sciences, vol. 545, no. 1, pp. 465-486, 2021.

[25] H. R. Medeiros, F. D. Oliveira, H. F. Bassani, and A. Araujo, "Dynamic topology and relevance learning SOM-based algorithm for image clustering tasks," Computer Vision and Image Understanding, vol. 179, no. FEB, pp. 19-30, 2019.

[26] Y. Deng, D. Huang, S. Du, G. Li, and J. Lv, "A double-layer attention based adversarial network for partial transfer learning in machinery fault diagnosis," Computers in Industry, vol. 127, Article ID 103399, 2021.

[27] J. J. Chan, K. K. Chen, and S. Sarker, "Epidemiology of Achilles tendon injuries in collegiate level athletes in the United States," International Orthopaedics, vol. 44, no. 3, pp. 585-594, 2020.

[28] W. Li, G. G. Wang, and A. H. Gandomi, "A survey of learningbased intelligent optimization algorithms," Archives of Computational Methods in Engineering, pp. 1-19, 2021.

[29] G. G. Wang, A. H. Gandomi, and A. H. Alavi, "A comprehensive review of krill herd algorithm: variants, hybrids and applications," Artificial Intelligence Review, vol. 51, no. 1, pp. 119-148, 2019. 